\title{
UNCERTAINTY QUANTIFICATION THROUGH A MODEL-BASED FUZZY SET MEMBERSHIP FUNCTION
}

\author{
J. Chleboun*
}

\begin{abstract}
An approach to uncertainty quantification is proposed. It is based on a fuzzy set membership function that is defined through a model (or models) response. That is, information about uncertain parameters is gathered from measured responses of one or more models, the membership function is inferred and used in uncertainty quantification of the output of a model of interest. The idea is illustrated by a beam deflection problem.
\end{abstract}

Keywords: Uncertainty quantification, fuzzy parameter, membership function, measurements, beam

\section{Introduction}

In uncertainty quantification based on fuzzy set theory, crisp quantities are replaced by fuzzy quantities, that is, by fuzzy sets. Each fuzzy set can be identified with its membership function representing the membership grade of the elements of the fuzzy set, see Dubois and Prade (2000), Möller and Beer (2010), or Zimmermann (2001), for instance. A simple but commonly used membership function has the range of $[0,1]$ and a triangular graph, see Fig. 1 (left). Then, the fuzzy set is defined as the support of the membership function or as the closure of the support. The latter approach is used in this contribution.

\subsection{Elements of fuzzy set theory}

To avoid mathematically interesting but computationally difficult problems, we assume that $S \subset V$ is an arc-connected compact and convex fuzzy subset of a normed space $V$ and $\mu_{S}$ is a continuous and concave membership function defined on $S$ and with range $[0,1]$ and such that $S$ is the closure of supp $\mu_{S}$. The function $\mu_{S}$ gives rise to a family of convex compact sets $S^{\alpha}$, the $\alpha$-controlled subsets of $S$ called $\alpha$-cuts or $\alpha$-level sets and defined as follows

$$
S^{\alpha}=\left\{x \in S \mid \mu_{S}(x) \geq \alpha\right\}, \text { where } \alpha \in(0,1] \text { and } S^{0} \equiv S .
$$

By knowing the set of all $\alpha$-cuts $S^{\alpha}$, we can reconstruct the membership function

$$
\mu_{S}(x)=\sup \left\{\alpha \mid x \in S^{\alpha}\right\} \quad \text { at } x \in S .
$$

The relation (2) is the tool for the analysis of uncertainty propagation through a mathematical model. Indeed, let $\Psi: S \rightarrow \mathbb{R}$, where $\mathbb{R}$ stands for the field of real numbers, be the input-to-output map representing the response of a quantity of interest to input data processed by a mathematical model. Let us assume that $\Psi$ is continuous. The range of $\Psi$ is a fuzzy set $S_{\Psi}$ with the membership function determined by Zadeh's extension principle, see Dubois and Prade (2000), Möller and Beer (2010), or Zimmermann (2001),

$$
\mu_{S_{\Psi}}(y)=\sup _{\{x \in S \mid y=\Psi(x)\}} \mu_{S}(x) \text { at } y \in S_{\Psi} .
$$

Jan Chleboun: Department of Mathematics, Faculty of Civil Engineering, Czech Technical University in Prague, Thákurova 7; 166 29, Prague 6; CZ, jan.chleboun@cvut.cz 

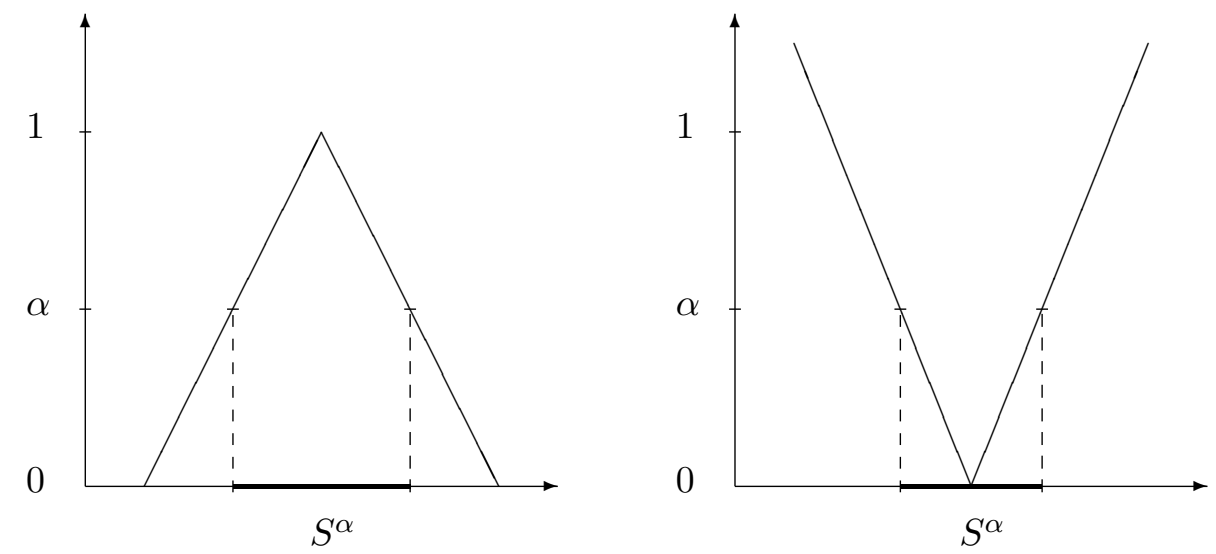

Fig. 1: A membership function and an $\alpha$-level set (left). An "upside down membership function" and an $\alpha$-level set (right).

Remark 1: By virtue of the assumptions, the supremum can be replaced by the maximum in (2) and (3).

The $\Psi$-image of each $S^{\alpha}$ is a closed interval

$$
S_{\Psi}^{\alpha}=\left[\min _{x \in S^{\alpha}} \Psi(x), \max _{x \in S^{\alpha}} \Psi(x)\right] .
$$

Indeed, if $y \in S_{\Psi}^{\alpha}$, then $x \in S^{\alpha}$ exists such that $y=\Psi(x)$. That is, $S_{\Psi}^{\alpha}$ is a subset of the $\Psi$-image of $S^{\alpha}$. If $x \in S^{\alpha}$, then $\Psi(x) \in S_{\Psi}^{\alpha}$, and we conclude that $S_{\Psi}^{\alpha}=\Psi\left(S^{\alpha}\right)$. By combining this conclusion, Remark 1, the principle (3), and the definition (1) with $S_{\Psi}$ and $\mu_{S_{\Psi}}$ replacing $S$ and $\mu_{S}$, we infer that $S_{\Psi}^{\alpha}$ is the $\alpha$-level set of $S_{\Psi}$ determined by $\mu_{S_{\Psi}}$. We can approximate the membership function $\mu_{S_{\Psi}}$ through solving a sequence of minimization and maximization problems (4) for a sequence of values $\alpha$, see also Möller and Beer (2010) and the next sections.

\section{Construction of the membership function}

In most cases, the membership function is triangular or trapezoidal and is defined on the basis of expert knowledge of the fuzzy quantity. In some situations, however, a less subjective approach is possible. To fix ideas, let us imagine a quantity of interest $\Psi(p) \in \mathbb{R}$ depending on a parameter $p$ that is not known exactly. Moreover, let $p$ appear as a parameter in another model, denoted $m_{p}$, whose output is measured. That is, a set $M=\left\{v_{i}\right\}_{i=1}^{n}$ of measured values is available and represents the behavior of $m_{p}$ for a fixed $p$. The measurements are burdened with errors. In the standard approach, the (weighted) least squares method is applied to $M$ and $m_{p}$ to identify $p_{\min }$, the crisp value of $p$ that implies the best agreement between the response of $m_{p}$ and the data $M$. Consequently, the identified parameter $p_{\min }$ is employed in the model of interest and its output $\Psi\left(p_{\min }\right)$ is calculated as a crisp quantity.

Due to the measurement errors, the parameter $p_{\min }$ is uncertain and the uncertainty quantification of $\Psi(p)$ attracts our attention.

If, for $i=1, \ldots, n$, we introduce $m_{p}^{i}$, the calculated response of the model $m_{p}$, and $w_{i}>0$, the weights associated with the data, then $p_{\min }$ minimizes the objective function

$$
f(p)=\sum_{i=1}^{n} w_{i}\left(v_{i}-m_{p}^{i}\right)^{2}
$$

over $S$, a set of admissible parameters. It is assumed that $f\left(p_{\min }\right)>0$.

To fuzzify $p$, let

$$
\mu_{1}(p)=c\left(\frac{f\left(p_{\min }\right)}{f(p)}-1\right)+1, \quad p \in S, c \in \mathbb{R}, c>0,
$$

and consider a fuzzy set $S_{1}=\left\{p \in S \mid \mu_{1}(p) \in[0,1]\right\}$. The set $S_{1}$ is also controlled by the fixed constant $c$. 
Remark 2: The definition (6) is not the only option. We can, for example, define

$$
\mu_{2}(p)=2-\frac{f(p)}{f\left(p_{\min }\right)}
$$

and consider only $p \in S$ such that $f(p) \leq 2 f\left(p_{\min }\right)$. In the uncertainty propagation analysis, it can be convenient to use an "upside down membership function"; see Figure 1 (right) and Ben-Haim (2006), where this idea is applied. In this concept, $\alpha$ is not limited to $[0,1]$, but the definition of $\alpha$-cuts remain unchanged except for the direction of the inequality in (1). As an example, take

$$
\mu_{3}(p)=\frac{f(p)}{f\left(p_{\min }\right)}-1, \quad p \in S .
$$

Unlike the original concept, increasing $\alpha$ increases the width of the $\alpha$-cut in the "upside down" approach. That is, the larger $\alpha$ is, the more uncertainty is taken into account at the $\alpha$-level.

By means of $\mu_{1}, \mu_{2}$, or $\mu_{3}$, the uncertainty in the parameter $p$ is quantified. The goal is then to quantify the uncertainty in $\Psi$, the quantity of interest. To this end, the intervals (4) are inferred and the membership function $\mu_{S_{\psi}}$ is constructed through (2), where $S$ and $S^{\alpha}$ are replaced by $S_{\Psi}$ and $S_{\Psi}^{\alpha}$, respectively.

\section{Beam deflection example}

Let us consider a beam of length $L=2$ in three different situations (a)-(c).

The deflection of the simply supported beam is measured at $L / 2$ (a) once under the concentrated central load $P=20000$ and (b) twice under the concentrated asymmetric load of the same magnitude and direction acting at the point $L-b$ where $0.15 L \leq b \leq 0.3 L$. The measured deflections are $v_{1}=0.0810$, and $v_{2}=0.0558, v_{3}=0.0593$, respectively. The quantity of interest is defined as (c) the deflection of the cantilever beam at the free end, where the point load $P$ is applied again.

As already indicated, the asymmetric load is applied at the point determined by the uncertain parameter $b$. Also, the product of $E$, the elastic modulus, and $I$, the second moment of area of the beam's cross-section, is not known exactly. For simplicity, let us introduce $a \equiv E I$. The parameter $p$ (see Section 2) then represents the pair $(a, b) \in U$, where $U$ is a set of admissible parameters, take $U=[30000,60000] \times[0.3,0.6]$, for instance.

According to Wikipedia (2018), the respective deflections are given by

$$
w_{\text {supported }}(a, b)=\frac{P b\left(3 L^{2}-4 b^{2}\right)}{48 E I}, \quad \Psi(a, b) \equiv w_{\text {cantilever }}(a, b)=\frac{P L^{3}}{3 E I},
$$

where $w_{\text {supported }}$ is the response at $L / 2\left(b=L / 2\right.$ in the case of the central load) and $\Psi \equiv w_{\text {cantilever }}$ is the response of the cantilever beam at $L$.

The function (5) reads:

$$
f(a, b)=\left(v_{1}-w_{\text {supported }}(a, L / 2)\right)^{2}+\frac{1}{2} \sum_{i=2}^{3}\left(v_{i}-w_{\text {supported }}(a, b)\right)^{2} .
$$

The minimum of $f$ is attained at $a_{\min }, b_{\min }$ and, moreover, $f\left(a_{\min }, b_{\min }\right)=1.7699 \times 10^{-3}$.

Let $\mu$ be given by (7), that is,

$$
\mu(a, b)=2-\frac{f(a, b)}{f\left(a_{\min }, b_{\min }\right)},
$$

and $S=\{(a, b) \in U \mid \mu(a, b) \geq 0\}$.

To obtain the intervals $S_{\Psi}^{\alpha}=\left[l_{\Psi}^{\alpha}, r_{\Psi}^{\alpha}\right]$, see (4), the following minimization and maximization problems have to be solved:

$$
l_{\Psi}^{\alpha}=\min _{\{(a, b) \in U \mid \mu(a, b) \geq \alpha\}} \Psi(a, b), \quad r_{\Psi}^{\alpha}=\max _{\{(a, b) \in U \mid \mu(a, b) \geq \alpha\}} \Psi(a, b)
$$

for $\alpha \in[0,1]$. The graph of the membership function associated with the fuzzy quantity of interest is then formed by points with the coordinates $\left(l_{\Psi}^{\alpha}, \alpha\right)$ and $\left(r_{\Psi}^{\alpha}, \alpha\right)$. 
The problems (11) were solved in the Matlab ${ }^{\circledR}$ environment with the help of the Optimization Toolbox ${ }^{\mathrm{TM}}$ function fmincon. The function was used to minimize $\Psi(a, b)$ (or $-\Psi(a, b)$ in the maximization problem) under the constraint $\mu(a, b) \geq \alpha_{k}$, where $\alpha_{k}, k=0,1, \ldots, m$, are values uniformly distributed over the interval $[0,1]$ and $\alpha_{0}=0, \alpha_{m}=1$. Thus, the membership function of the quantity of interest is approximated, see Figure 2. The least squares problem was solved by the fminsearch function.

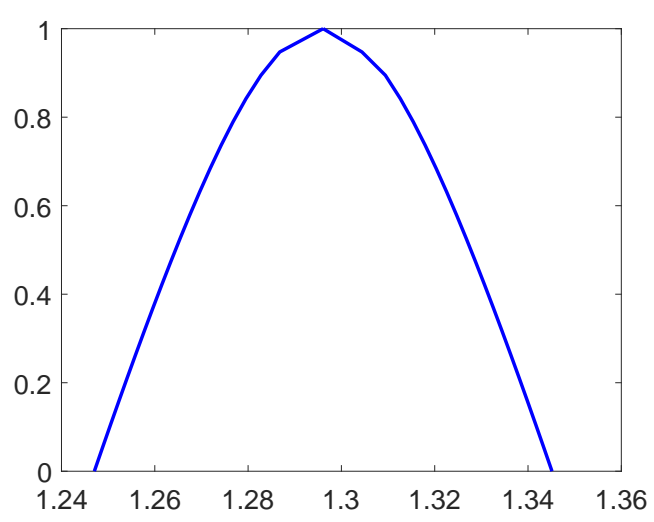

Fig. 2: The membership function inferred from the endpoints $l_{\Psi}^{\alpha}$ and $r_{\Psi}^{\alpha}$, see (11). The horizontal axis corresponds to $w_{\text {cantilever, }}$ see (9).

\section{Conclusions}

The presented method of determination of the membership function of fuzzy input data reduces the need for analyst intervention. The membership function is inferred directly from the measurements. Nevertheless, the analyst is responsible for choosing the weights in the weighted least squares and the proper form of the input membership function. The examples (6)-(8) are not exhaustive and other expressions can be proposed. Also, square root can be considered in (5).

\section{Acknowledgments}

The author's work was funded by the Czech Science Foundation through grant No. 18-04262S. The author is grateful to Dr. Richard (Dick) Haas for many fruitful discussions.

\section{References}

Ben-Haim, Y. (2006), Info-Gap Decision Theory; Decisions Under Severe Uncertainty, 2nd edition, Elsevier, Amsterdam.

Dubois, D., Prade, H. (2000), Fundamentals of fuzzy sets, The Handbooks of Fuzzy Sets Series, Vol 7, Kluwer Academic Publishers, Dordrecht.

Möller, B., Beer, M. (2000), Fuzzy Randomness; Uncertainty in Civil Engineering and Computational Mechanics, Springer-Verlag, Berlin.

Wikipedia(2018), https://en.wikipedia.org/wiki/Euler\%E2\%80\%93Bernoulli_beam_theory, visited on January 9, 2018.

Zimmermann, H.-J. (2001), Fuzzy Set Theory - and Its Applications, 4th edition, Kluwer Academic Publishers, Boston. 